\title{
Ciencia básica, ciencia aplicada, extensión y divulgación: de la compartimentalización a la ecología de saberes o transdisciplinariedad
}

Basic science, applied science, extension and scientific divulgation: from compartmentalization to ecology of knowledge or transdisciplinarity

María Julieta Duedra

https://orcid.org/0000-0002-1584-2900

julieta.duedra@gmail.com

Facultad de Ciencias Jurídicas y Sociales |

Universidad Nacional de La Plata | Argentina

Martin Miguel Montes

https://orcid.org/0000-0002-7177-333X

martinmiguelmontes@gmail.com

Facultad de Ciencias Naturales y Museo I

Universidad Nacional de La Plata | Argentina

\author{
Paula Gabriela Tobes \\ https://orcid.org/0000-0002-7450-8582 \\ paula.tobes@gmail.com \\ Facultad de Ciencias Juridicas y Sociales | \\ Universidad Nacional de La Plata | Argentina
}

Fernando Galliari

https://orcid.org/0000-0002-2659-7373

fgalliari@gmail.com

División Paleontología de Vertebrados | Museo

de La Plata | Facultad de Ciencias Naturales y

Museo | Universidad Nacional de La Plata |

Argentina.

\section{RESUMEN}

En el presente trabajo se expone nuestra visión de la ciencia básica, aplicada y del rol de ambas en nuestra sociedad. Se explicitan algunas falencias del sistema científico e institucional que podrían conllevar a la confrontación de estos dos campos, en apariencia separados. Además, se analiza el rol de la extensión universitaria en la ciencia aplicada, extensión articulación entre las ciencias básica y aplicada, los diferentes elementos que intervienen en la interacción entre ambas y las dificultades de dicho proceso.

\section{ABSTRACT}

The aim of this paper is to present our vision on basic and applied science, and the role that they have in our society. Some flaws of the scientific and institutional systems are explicit that may explain the confrontation of these two apparently separated fields. Furthermore, this work analyzes the role of the university extension in

\section{KEY WORDS}

basic science, applied science, extension the articulation between basic and applied sciences, the different elements involved in this interaction and the difficulties of this process. 


\section{CIENCIA BÁSICA VS CIENCIA APLICADA}

Últimamente en la ciencia existe una disputa generada entre la llamada "ciencia básica" vs "ciencia aplicada". Reduciendo un poco sus definiciones se podría decir que la ciencia básica considera que el conocimiento tiene derecho de existir por motus propio, mientras que la ciencia aplicada sirve como base a un aprovechamiento para la sociedad.

\section{la ciencia básica considera que el conocimiento tiene derecho de existir por motus propio, mientras que la ciencia aplicada sirve como base a un aprovechamiento para la sociedad.}

Cuando el neoliberalismo se introduce dentro de la ciencia e intenta avasallarla para conseguir más renta, se potencian dificultades y rivalidades y vemos que podemos encontrar que estas definiciones son más antiguas. Empero, algunos de nosotrxs creemos que esta dualidad se hunde en la historia misma de la humanidad, mientras que otrxs pensamos que podemos remontarnos hacia un pasado más cercano y concreto y encontrar que la filosofía de las ciencias puede ayudarnos un poco a comprender esta disputa.

Históricamente en el surgimiento de los diferentes movimientos de filósofos de la ciencia se ven diferentes líneas de pensamiento, como la de "concepción heredada" (inductivistas, hipotético inductivistas, falsacionistas Popperianos), donde la producción del conocimiento es independiente de las influencias externas -presiones sociales, culturales, entre otras-. Lxs seguidores de esta ideología creen que la ciencia debe seguir su propia lógica y luchar por independizarse de las interacciones sociales, políticas y económicas. Para ellxs la objetividad está ligada al método, el cual es histórico y hegemónico en el sentido que sujeto, verdad, objetividad, y método están libres de todo factor socio-cultural. 


\section{"concepción heredada" (inductivistas, hipotético inducti- vistas, falsacionistas Popperianos), donde la producción del conocimiento es independiente de las influencias externas -presiones sociales, culturales, entre otras-.}

Esta forma de pensar es la que ha quedado en el imaginario de la sociedad -y varixs científicxs- y se relaciona estrechamente con la concepción que tenemos de ciencia básica. Algo aséptico, sin ningún tinte político, ni social, sólo interesándose en el conocimiento y no importando lo que deriva de él. Esta forma de ver la ciencia representa a Ixs científicxs como "seres cuya única ambición es alcanzar la verdad, hacer avanzar la frontera del conocimiento" (Andrini y Liaudat, 2019); seres recluidxs, introvertidxs, dueñxs de una gran inteligencia pero ingenuxs, una especie de autistas sin contacto con otrxs seres humanos, olvidándose de las relaciones personales y del tacto social, entre otras cuestiones importantes, viviendo encerradxs en sus laboratorios. Esta visión del científicx y de la ciencia tiene otra arista, mencionada por Andrini y Liaudat (2019) cuando hablan del "fetichismo por la ciencia". Una especie de mirada sacra por parte de la sociedad atribuyéndole una posición privilegiada a la ciencia junto a sus actores -Ixs científicxscon sus títulos, cargos, membresías, etc. Sin embargo, esta concepción de la ciencia y Ixs científicxs podría ser rebatida cuando vemos, en la actualidad, la fascinación que produce un/a youtuber, instagramer, o deportista en contraposición a un/a científicx. Cuando se analiza el reconocimiento económico que recibe cada unx, o en relación a otros estamentos del Estado -comparando años de formación y salario- se percibe una gran diferencia. Formar un/a científicx le consume al Estado fondos y a la persona aproximadamente 12 años de formación -tomando como base la formación del nivel secundario- los cuales se reparten del siguiente modo: $5 / 6$ años de la carrera universitaria de grado, 5 años de postgrado y 2 años mas de perfeccionamiento postdoctoral en muchos casos. Este destrato o doble vara para medir al científicx ha sido más palpable en el contexto de pandemia que estamos atravesando por causa del COVID-19, en situaciones donde un/a modelo de televisión aconseja "medicamentos" para combatir el COVID-19 o un presidente sin formación en medicina aconseja tal o cual medicamento -en muchos casos desaconsejados por la Organización Mundial de la Salud-, mientras que Ixs verdaderxs profesionales y organismos especializados son ignorados, menospreciados y en algunos casos cuestionados por algunos sectores de la sociedad. En este sentido, Andrini y Liaudat (2019) mencionan la dualidad presente en la sociedad sobre considerar a la ciencia como algo bueno o malo, ¿es algo que salvará a la humanidad, o el advenimiento de, por ejemplo, una inteligencia artificial que nos eliminará?. Recurriendo a ejemplos 
actuales, existen personas convencidas de que la ciencia es quien producirá la vacuna para combatir el COVID-19 mientras otrxs postulan que fue la misma ciencia quien generó la presente pandemia.

\section{Esta forma de ver la ciencia representa a Ixs científicxs como "seres cuya única ambición es alcanzar la verdad, hacer avan- zar la frontera del conocimiento"}

Como contraposición a esa filosofía que deshumaniza la ciencia, existe otra vertiente que reivindica lo social y lo histórico, criticando esta racionalidad preestablecida, universal, independiente del contexto y de las circunstancias históricas. Ejemplos de filósofos de esta línea de pensamiento son Lakatos y Kuhn, este último plantea que las comunidades científicas son principalmente conservadoras y dogmáticas. Los científicxs son seres sociales, se reúnen y comunican sus descubrimientos por medio de publicaciones, pero sólo escriben para sus colegas y no para el público general, siendo ellxs mismxs público y juez/a de sus actividades, regulando quién es aceptadx o no en su propio seno. Latour plantea una doble existencia en todo grupo, por un lado una social y por otro una cognitiva. Esta doble existencia también está presente en el paradigma de cada rama de la ciencia y a su vez, la indisociabilidad de lo social y lo cognitivo hace que el grupo no pueda definirse por fuera de las concepciones del mundo que sus miembros comparten y que estructuran los conocimientos que aquel produce. Esta forma de pensar del científicx como parte de la estructura social da origen a un tipo de investigador/a que no sólo surge como parte de la sociedad que representa, sino que está marcadx por los acontecimientos históricos, y como parte de ellos intenta realizar algún aporte (Prebble, 2020). Esta concepción filosófica se observa con mayor interés por parte de Ixs científicxs dando origen al surgimiento de una ciencia más aplicada.

\section{Como contraposición a esa filosofía que deshumaniza la cien- cia, existe otra vertiente que reivindica lo social y lo histórico, criticando esta racionalidad preestablecida, universal, inde- pendiente del contexto y de las circunstancias históricas.}

La ciencia básica fue considerada por mucho tiempo como un gasto para el Estado -especialmente por los gobiernos de neoliberal-y no como una inversión necesaria, lo cual se vió reflejado en acciones de recorte presupuestario, trabas burocráticas o bien, en muchos casos, 
la eliminación de ministerios abocados al desarrollo científico como el Ministerio de Ciencia y Tecnología e Innovación-. Lxs científicxs dedicados a cuestiones básicas se encuentran también con otros escollos: la escasa adjudicación de subsidios y la falta de interés de invertir en ella, basándose en que no existe una aplicabilidad de tales conocimientos en el corto plazo. Además de la concepción política de ello, la misma sociedad es quien no reconoce a la ciencia básica como algo útil que reditúe en algún modo a su bienestar. Y esto, realmente se presenta como una cuestión problemática, es decir, parecería restringir la producción de conocimiento a lo "útil" -para quien financia- e inmediato, sin tener en cuenta quizás otras cuestiones más vinculadas con la prevención a largo plazo o las áreas relegadas por falta de interés, pero que, no por ello son menos importantes. También es verdad que muchas veces la ciencia avanza sin que Ixs actores involucradxs se detengan a pensar ni dejar que la sociedad sea consciente, evalúe y apruebe si un determinado avance es correcto éticamente o necesario (Andrini y Liaudat, 2019). Por esta razón es importante que las personas dedicadas a la ciencia tomen "con-ciencia" -si se permite el juego de palabras- de la necesidad de comunicar a la sociedad el producto de sus investigaciones e intentar, mediante su pericia, conocer y solucionar los problemas sociales.

\section{muchas veces la ciencia avanza sin que Ixs actores involu- cradxs se detengan a pensar ni dejar que la sociedad sea consciente, evalúe y apruebe si un determinado avance es correcto éticamente o necesario}

Además de los problemas con la obtención de subsidios y poca valoración política y social, la ciencia -y no solamente la básica- se enfrenta a la "inmediatez" exigida a los trabajos científicos que, en muchos casos, obliga a compartimentalizar el conocimiento bajo el riesgo de perder cohesión y sentido de los contenidos y con el solo fin de cumplir con las demandas del sistema científico. Tales hechos terminan estableciendo otras prioridades que van más allá de las que unx considera adecuadas: antes que calidad, cantidad, y antes que cantidad, aplicabilidad.

Otra cuestión problemática es "quién" o en base "a qué" consideramos "buena ciencia" o si los aportes realizados por las publicaciones son realmente importantes. Un ejemplo concreto lo vemos en la forma de evaluar -a sus científicos- que aplica el Consejo Nacional de Investigaciones Científicas y Técnicas (CONICET), que considera revistas especializadas aquellas que figuran en SCImago Journal \& Country Rank. La mayoría de ellas son de habla inglesa, lo cual obliga a científicxs a 
tener que aprender ese idioma para poder publicar. También implica un gasto que podría evitarse ya que muchxs recurren a los propios subsidios del Estado para poder pagar el costo de publicar -en dólares- y en pagar traducciones de nativxs de lengua inglesa dado que así lo demanda la revista. En muchos casos se reciben observaciones/ sugerencias de las revistas solicitando mejorar el inglés - debido a que es poco claro- o bien terminan por ofrecer un servicio -pago- de edición a cargo de su propio equipo editorial. Este gasto de subsidio -si se tiene la suerte de contar con alguno, o si se lo reintegra en tiempo y forma porque la inflación y la demora en hacerse efectivo el depósito del mismo termina desvalorizando el presupuesto original- en publicación y traducción se hace para que el CONICET considere "aptos" a sus científicos, independientemente de la calidad del trabajo o su aplicabilidad dentro del país. Dicha problemática se profundiza, por ejemplo, en casos en los cuales este tipo de revistas especializadas -muchas veces- devuelven los trabajos por considerarlos de "importancia local" o"regional". Es posible que esto sea así, aunque no debería serlo, puesto que hay problemas que surgen de forma nacional, local o regional y se extienden hacia otras partes del continente o incluso al mundo. Un ejemplo de ello es el COVID-19 que nació como algo local o regional, y sin embargo se globalizó rápidamente, lo mismo podría -si no está sucediendo en estos momentos- con la aparición del virus Chapare en Bolivia que se ha mencionado últimamente en los periódicos nacionales y se alarmaba sobre su transmisión de persona a persona. Esta desacreditación a un descubrimiento regional o local es algo a replantearse por parte de las autoridades del CONICET y quienes tenemos la posibilidad de evaluar papers con investigaciones "regionales o locales" ya que hacen al concepto de soberanía científica que mencionan Andrini y Liaudat (2019).

Todo esto nos lleva a pensar en que se ha generado un negocio en torno a las publicaciones, donde Ixs científicxs son una especie de "jornalerx de campo" y la información no llega a la gente que puede tomar decisiones, y/o al público en general que son quienes, indirectamente, aportan dinero a través de los impuestos y que, en definitiva, se encuentran manteniendo nuestros estudios y profesiones. Mientras el CONICET no haga un cambio en la forma de evaluar a sus científicxs, de apoyar revistas locales o regionales, no tendremos una ciencia soberana y se continuará investigando según la agenda que establezcan los países centrales.

Las revistas que son consideradas de élite y que se exigen a lxs científicxs para que publiquen se basan, en la actualidad, como mencionamos, en la clasificación del portal SCImago, pero antes el indicador más importante era el Factor de Impacto (FI), aún usado en el mundo, que fue calculado originalmente por Thomson Reuters como una herramienta para ayudar a Ixs bibliotecarixs a identificar qué revistas 
comprar y no era, como se entiende ahora, una medida de la calidad científica de la investigación de un artículo (Moustafa, 2014). Las limitaciones del FI son varias: las distribuciones de citas dentro de las revistas están muy sesgadas; las propiedades del FI son específicas de campo, es un compuesto de múltiples tipos de artículos muy diversos que incluyen artículos de investigación y revisiones; puede ser manipulado o controlado por la política editorial; los datos utilizados para calcular los Fl de la revista no son transparentes ni están públicamente disponibles (ver Declaración de San Francisco sobre la Evaluación de la Investigación (DORA), https://sfdora.org/read/es/).

Desde el año 2011, como bien dice en su página, surgió Sci-hub, bajo una idea realmente revolucionaria "La distribución de artículos de investigación, como de otras fuentes científicas o educacionales, está artificialmente -aunque legalmente- restringida por leyes de derechos de autor. Esas leyes retrasan el desarrollo de la ciencia en la sociedad. Sci-hub está cambiando el status quo, evitando cualquier pago y restricciones" Esta página se erige sobre 3 ideas básicas: Conocimiento para todos, no hay derechos de autor ni propiedad intelectual, y por último el acceso abierto en ciencia.

Sin lugar a dudas la reacción de la comunidad científica frente a este factor de impacto, la poca reacción del CONICET para defender la soberanía de la ciencia fomentando la publicación en revistas indexadas del exterior nos lleva indefectiblemente a analizar las sugerencias que hace la Declaración de San Francisco sobre la Evaluación de la Investigación (DORA). En su declaración postula que "los productos que no sean artículos de investigación crecerán en importancia a la hora de evaluar la eficacia de la investigación en el futuro, pero el documento de investigación revisado por pares seguirá siendo primordial para la evaluación de la investigación". Las sugerencias se aplican, no solo a los artículos de investigación, sino a los artículos de otro tipo y plantean como base que no se utilicen las métricas basadas en revistas -como el Fl-, sino que se sustituya por la calidad de los artículos de investigación individuales para evaluar las contribuciones de un/a científicx individual o en las decisiones de contratación, promoción o financiación. Posteriormente, se explaya sobre recomendaciones para las agencias de financiación, instituciones, editoriales, organizaciones que proporcionan métricas, y para Ixs investigadores. En este sentido, solo haremos una pequeña referencia en cuanto a las instituciones y lxs investigadorxs. Para las primeras se sugiere que sean explícitas en los criterios usados para la contratación, permanencia y promoción dando más valor a la calidad del trabajo que al lugar donde ha sido publicado -editorial, revista, etc.-; que se considere el valor y el impacto de todos los resultados de la investigación, no solo las publicaciones de investigación sino que incluya indicadores cualitativos como su influencia sobre la política y prácticas científicas. Para Ixs investigadorxs sugiere que al participar de comités que tomen 
decisiones sobre financiamiento, contratación, permanencia o promoción se realice una evaluación basada en el contenido científico y no en el lugar de publicación de sus artículos; cuando sea posible se cite la literatura primaria en que las observaciones son referidas en primer lugar -antes que a las revisiones- para dar crédito donde debe darse -esto es muy importante en casos donde la información de base surge justamente de publicaciones entendidas como de "ciencia básica"-, utilice una gama de indicadores basadas en declaraciones personales y de apoyo como evidencia del impacto de artículos individuales publicados y otros resultados de investigación, impugne las prácticas de evaluación que dependen indebidamente del Fl y promueva y enseñe prácticas que se centren en el valor y la influencia de los resultados de investigación específicos.

A partir de lo establecido por Andrini y Liaudat (2019), las sugerencias de DORA y de Sci-hub, nos planteamos ¿para quién investiga el/ la científicx? ¿Son -somos- Ixs científicxs tal como dice Andrini y Liaudat (2019) seres con esa aura de "genialidad, incomprensión, desinterés, aislamiento, superioridad, clarividencia, tenacidad, determinación, cosmopolitismo y comunitarismo entre otras cualidades"? ¿somos tan "opacos" que "menospreciamos el aspecto social de la construcción de conocimiento, las relaciones sociales implicadas, las múltiples interacciones y negociaciones involucradas"? ¿La ciencia es única y universal? ¿O como bien dice el artículo, "el aparato cultural de las grandes potencias es el que alimenta los esfuerzos científicos en el mundo entero de acuerdo a una agenda, temas de investigación, metodologías y lenguajes que sirven sólo a las potencias mundiales"? ¿Cómo es posible que nuestros descubrimientos lleguen a la sociedad o a la gente que toma decisiones si nos vemos forzadxs a publicar en revistas internacionales, bajo estándares de otros países y en otros idiomas? Y es en la respuesta de esta pregunta desde donde debimos empezar este artículo. En parte ya se explicó y presentó el problema en los párrafos anteriores, pero por más que como científicxs quisiéramos llegar al público en general, salteando los problemas institucionales, nos separa de la sociedad la forma que tenemos de relacionarnos, nuestras experiencias y conocimientos. Nuestro trabajo debe ser comunicado de forma amena al resto de la comunidad en entornos cuidados y por gente con experiencia en esa forma de comunicación, de aquí surge la necesidad de que la extensión y la divulgación sean una construcción multidisciplinaria.

\section{Nuestro trabajo debe ser comunicado de forma amena al res- to de la comunidad en entornos cuidados y por gente con experiencia en esa forma de comunicación, de aquí surge la necesidad de que la extensión y la divulgación sean una cons- trucción multidisciplinaria.}


¿Cómo trasladar el producto de las investigaciones a proyectos de extensión? Desafíos y dificultades.

El proceso de construcción de conocimiento y aplicación de saberes teóricos a nuestra práctica se presenta, en muchos casos como una cuestión problemática puesto que implica la intervención de diferentes conceptos tales como: la ecología de saberes y la transdisciplinariedad, que para que sean viables es necesario previamente derribar obstáculos estructurales y personales que tienen que ver con la construcción misma del campo disciplinar. Según Boaventura de Sousa Santos (2007), la cuestión primordial está en saber vincular los campos, en adoptar herramientas de todos ellos y es aquí donde reside realmente el desafío que no dejamos de ver como algo complejo pero que es necesario que se supere.

El proceso de construcción de conocimiento y aplicación de saberes teóricos a nuestra práctica se presenta, en muchos casos como una cuestión problemática puesto que implica la intervención de diferentes conceptos tales como: la ecología de saberes y la transdisciplinariedad

En este proceso la extensión juega un papel fundamental como articulador, aunque posea poco peso en el contexto de las universidades dado que, en muchos casos, queda relegada a actividades secundarias de las cuales se tiene poco conocimiento, y/o que las trabas burocráticas generan un retraso en la concreción de sus proyectos. No obstante ello, es importante que la extensión asuma un rol activo en lo que respecta a las problemáticas sociales, políticas y económicas, en donde se interactúe más con las comunidades en las que se desarrolla y también en la divulgación de sus actividades para permitir el conocimiento de sus mecanismos y actuaciones.

\section{extensión juega un papel fundamental como articulador}

El objetivo central al cual se podría aspirar en esta articulación es la generación de proyectos que sean asumidos por toda la "comunidad universitaria" como integradora y abarcativa de las distintas disciplinas y ,por otra parte, incluir en la toma de decisiones a Ixs diferentes actores, puesto que la extensión implica tener en cuenta a quienes están involucradxs en la problemática que se aborde, sus intereses y subjetividades, dejando de lado la clásica unidireccionalidad que se presenta en la extensión.

Actualmente, el conocimiento científico es fraccionado en campos disciplinares que componen grupos de gestión del conocimiento, que 
se distinguen por tener un objeto particular de estudio y un saber especializado sobre este. Estos campos disciplinares en las instituciones universitarias se ven reflejados en las diversas unidades académicas que la conforman, razón por la que este fraccionamiento adquiere un papel trascendental luego en las formas de enseñanza y aprendizaje y en la manera de hacer investigación y extensión.

En efecto, Burton Clark explica que se trata de "paquetes de conocimiento" que tienen cada uno sus propias características, no sólo en la enseñanza y la investigación, sino también en las prácticas. Por esta razón, resulta primordial implementar prácticas integrales que, según el Fascículo № 10"Hacia la Reforma Universitaria”, dentro de las características que las definen se encuentran, justamente, "a) la integración de los procesos de enseñanza y creación de conocimientos a experiencias de extensión; b) la perspectiva interdisciplinaria tanto en el nivel epistemológico vinculado a la enseñanza -tratamiento de los contenidos- y a la creación de conocimiento -construcción del objeto de investigación-, como en el nivel de intervención -construcción y abordaje de los problemas y conformación de los equipos...." (UR, Rectorado: 2010: 26)."(Cuadernos de Extensión, 1956, pág. 23 y 24).

La articulación de proyectos de extensión en cada Facultad que analizan y abordan la realidad desde una visión especializada y fragmentada no resulta suficiente para tratar fenómenos de gran complejidad que no es dable reducirlos en su tratamiento sólo a su aspecto teórico o práctico y únicamente por una sola disciplina, siendo merecedores de un abordaje multi, inter o transdisciplinario.

\section{La articulación de proyectos de extensión en cada Facultad que analizan y abordan la realidad desde una visión especia- lizada y fragmentada no resulta suficiente para tratar fenó- menos de gran complejidad que no es dable reducirlos en su tratamiento sólo a su aspecto teórico o práctico y únicamente por una sola disciplina, siendo merecedores de un abordaje multi, inter o transdisciplinario.}

Por ello, la propuesta emerge a partir de ponerse en evidencia la necesidad de implementar proyectos desde la Universidad que engloben, según la temática, los campos disciplinares propios de las unidades académicas que se encuentren involucradas en los proyectos de extensión y que permitan implementarlos y tratarlos desde su integralidad, dada la complejidad del mundo y, en particular, de la sociedad en la cual se van a instrumentar. Incluso, conforme menciona Boaventura de Sousa Santos y se aludió en forma precedente, en virtud de la "ecología de saberes", es dable y proficuo realizarlos desde el reconocimiento de otros saberes como los laicos, populares, tradicionales, es decir, considerando una ecología más amplia de saberes.

En este sentido, "un proyecto de extensión se concreta a través de un 
proceso de intervención social," que se realiza siempre en un "espacio cultural/social/histórico establecido y con un grupo de actores que tienen diferentes expectativas, motivaciones, intereses, tiempos y maneras de entender las cosas (Niremberg, 2006)." (Rafaghelli, M., 2013, pág. 28).

"Es un espacio social de aprendizaje y formación en tanto y en cuanto pueden convivir y buscar modos de articulación entre diferentes enfoques, creencias, culturas, expectativas e intereses." Los proyectos y actividades de extensión universitaria, como prácticas políticas y sociales, para que sean educativos deben posibilitar la construcción de un espacio de experiencias y saberes compartidos entre actores sociales con historias y culturas diferentes y con intereses y posibilidades desiguales. Una de las características que debe tener ese espacio es que debe ser interdisciplinario, es decir, conformado por profesionales de diversas áreas del conocimiento científico, con capacidades para promover instancias dialógicas y entender las problemáticas de los fenómenos que se trabajen. A su vez, debe ser un espacio de relaciones asimétricas donde operen actores sociales con biografías, culturas y roles diferentes desde los cuales cada uno aporta en el proyecto. (Rafaghelli, M., 2013, pág. 28).

Es clave internalizar y comprender que "estas propuestas suponen ante todo apartarse de los enfoques tradicionales," ya que "se privilegian formas de trabajo más exigentes y complejas, se trabaja con abordajes interdisciplinarios, se borran las fronteras de cada cátedra" y de cada unidad académica, las que se unen a pensar en conjunto. A través de la participación en este tipo de proyectos tanto alumnxs, como docentes, investigadorxs y graduadxs de diversas disciplinas"se enfrentan a la tarea de resolver problemas reales y a proponer mejoras en las comunidades y organizaciones donde se desarrollan los proyectos." (Torres y Trápaga, 2010:118)." (Rafaghelli, M., 2013, pág. 31 y 32).

\section{"estas propuestas suponen ante todo apartarse de los enfo- ques tradicionales," ya que "se privilegian formas de trabajo más exigentes y complejas, se trabaja con abordajes interdis- ciplinarios, se borran las fronteras de cada cátedra" y de cada unidad académica, las que se unen a pensar en conjunto.}

Volviendo a la discusión primigenia, en este periodo de la historia y encontrándonos en pleno aislamiento social, preventivo y obligatorio (ASPO) debido al COVID-19 las tecnologías digitales están jugando un papel predominante incrementando la velocidad y amplitud de la circulación de ideas, en la divulgación de noticias, formación, regulación de comportamiento y prácticas de la sociedad.

La universidad se está transformando paulatinamente, y considerando en adoptar herramientas virtuales a sus metodologías de enseñanza, pero aún no se ha planteado este tipo de interacción para la extensión. Una universidad democrática es aquella que da voz a todos Ixs actores de la sociedad. No todxs estos acceden a la universidad y en ese sentido 
no hay democracia universitaria posible en tanto no se democratice la posibilidad de acceder a la universidad para todxs ellxs.

Boaventura de Sousa Santos (2007) plantea que la universidad debe asumir el papel de articular la resistencia frente al neoliberalismo. Entre los requisitos que legitiman a la universidad como modelo de integración, especialmente después de la reforma de 1918, está la extensión que debe garantizar el vínculo de la misma con su contexto, el diálogo con el territorio donde se ancla no solo física, sino también identitariamente. A su vez, la extensión debe ser herramienta democratizadora de la universidad, vía de acceso de la palabra de quienes no acceden a sus aulas. De esta forma se busca que la Universidad ya no sea un espacio que implique solo "conocer sobre", sino también "conocer con". Tomando las palabras de Freire sobre la educación y aplicándolas a la extensión, "el conocimiento precisa de expresión y comunicación", es decir, se destaca la necesidad de una razón dialógica comunicativa, reconocer que los hechos de conocer y pensar están ligados a la relación con el otro.

\section{la extensión debe ser herramienta democratizadora de la uni- versidad, vía de acceso de la palabra de quienes no acceden a sus aulas. De esta forma se busca que la Universidad ya no sea un espacio que implique solo "conocer sobre", sino también "conocer con".}




\section{BIBLIOGRAFIA}

Andrini, L. y Liaudat, S. (2019). ¿Por qué discutir políticamente la ciencia y la tecnología?. En M. Speroni (coord.), “Política: apareces en el aire; dimensión misteriosa y escurridiza de la experiencia humana". En entredichos. Intervenciones y debates en Trabajo Social, Facultad de Trabajo Social, UNLP, Dossier Nº6.

Declaración de San Francisco sobre la Evaluación de la Investigación (DORA). 2012. https://sfdora.org/read/es/

De Sousa Santos, B (2007). La universidad en el siglo XXI. Para una reforma democrática y emancipadora de la universidad. México: UNAM / CEIICH. Capítulo 1, pp. 23-50.

"Misteriosa y escurridiza de la experiencia humana". En entredichos. Intervenciones y debates en Trabajo Social, Facultad de Trabajo Social, UNLP, Dossier $\mathrm{N}^{\circ} 6$.

Klimovsky, G (1994): Las desventuras del conocimiento científico, Buenos Aires, A-Z Editores.

Kuhn, T (1985): La Estructura de las Revoluciones Científicas, México, Fondo de Cultura Económica

Marí, E (1991): Ciencias y ética: el modelo de la ciencia martillo, en Doxa № 10, Universidad de Alicante.

Moustafa, K. (2014). The Disaster of the Impact Factor. Science and Engineering Ethics, 21(1), 139-142. doi:10.1007/s11948-014-9517-0

Prebble, M. 2020. Introducción al pensamiento científico. Cátedra Miguel. La nueva filosofía de la Ciencia. UBA CBC IPC. https:/www.youtube.com/watch?v=zKOwTfG6Dgl\&list=LLkdMqP6f3qPcmgxQnVyyikA\&index=3

Rafaghelli, M. (2013). La dimensión pedagógica de la extensión en Integración docencia y extensión. Otra forma de enseñar y de aprender Gustavo Menéndez et al. Universidad Nacional del Litoral. Santa Fe, Argentina.

Tommasino, H. y Rodriguez, N. (1956). Tres tesis básicas sobre extensión y prácticas integrales en la Universidad de la República en Cuadernos de Extensión - No 1 Integralidad: tensiones y perspectivas. ISSN : 1688-8324. Montevideo, Uruguay. 